\title{
Electronic properties of disordered graphene antidot lattices
}

\author{
Yuan, Shengjun; Roldán, Rafael; Jauho, Antti-Pekka; Katsnelson, M. I.
}

\section{Published in:}

Physical Review B Condensed Matter

Link to article, DOI:

10.1103/PhysRevB.87.085430

Publication date:

2013

Document Version

Publisher's PDF, also known as Version of record

Link back to DTU Orbit

Citation (APA):

Yuan, S., Roldán, R., Jauho, A-P., \& Katsnelson, M. I. (2013). Electronic properties of disordered graphene antidot lattices. Physical Review B Condensed Matter, 87(8), 085430.

https://doi.org/10.1103/PhysRevB.87.085430

\section{General rights}

Copyright and moral rights for the publications made accessible in the public portal are retained by the authors and/or other copyright owners and it is a condition of accessing publications that users recognise and abide by the legal requirements associated with these rights.

- Users may download and print one copy of any publication from the public portal for the purpose of private study or research.

- You may not further distribute the material or use it for any profit-making activity or commercial gain

- You may freely distribute the URL identifying the publication in the public portal

If you believe that this document breaches copyright please contact us providing details, and we will remove access to the work immediately and investigate your claim. 


\title{
Electronic properties of disordered graphene antidot lattices
}

\author{
Shengjun Yuan, ${ }^{1, *}$ Rafael Roldán, ${ }^{2, \dagger}$ Antti-Pekka Jauho, ${ }^{3}$ and M. I. Katsnelson ${ }^{1}$ \\ ${ }^{1}$ Radboud University of Nijmegen, Institute for Molecules and Materials, Heijendaalseweg 135, 6525 AJ Nijmegen, The Netherlands \\ ${ }^{2}$ Instituto de Ciencia de Materiales de Madrid, CSIC, Cantoblanco E28049 Madrid, Spain \\ ${ }^{3}$ Center for Nanostructured Graphene (CNG), DTU Nanotech, Department of Micro- and Nanotechnology, Technical University of Denmark, \\ DK-2800 Kongens Lyngby, Denmark
}

(Received 21 November 2012; revised manuscript received 15 January 2013; published 19 February 2013)

\begin{abstract}
Regular nanoscale perforations in graphene (graphene antidot lattices, GALs) are known to lead to a gap in the energy spectrum, thereby paving a possible way towards many applications. This theoretical prediction relies on a perfect placement of identical perforations, a situation not likely to occur in the laboratory. Here, we present a systematic study of the effects of disorder in GALs. We consider both geometric and chemical disorder, and evaluate the density of states as well as the optical conductivity of disordered GALs. The theoretical method is based on an efficient algorithm for solving the time-dependent Schrödinger equation in a tight-binding representation of the graphene sheet [Yuan et al., Phys. Rev. B 82, 115448 (2010)], which allows us to consider GALs consisting of $6400 \times 6400$ carbon atoms. The central conclusion for all kinds of disorder is that the gaps found for pristine GALs do survive at a considerable amount of disorder, but disappear for very strong disorder. Geometric disorder is more detrimental to gap formation than chemical disorder. The optical conductivity shows a low-energy tail below the pristine GAL band gap due to disorder-introduced transitions.
\end{abstract}

DOI: 10.1103/PhysRevB.87.085430

PACS number(s): 73.21.La, 72.80.Vp, 73.22.Pr

\section{INTRODUCTION}

Pristine graphene has no band gap: the conduction and valence bands touch at the $K$ and $K^{\prime}$ points of the hexagonal Brillouin zone. This property, combined with the linear dispersion of the low-energy excitations, leads to the spectacular electronic properties that graphene is so famous for. ${ }^{1,2}$ Nevertheless, the lack of a gap severely hampers many applications where a gap is needed to control the flow of charges. This feature is further underscored by the phenomenon of Klein tunneling: graphene carriers impinging on a potential barrier may experience reflectionless tunneling thus making their control even more difficult. ${ }^{3,4}$ It is thus natural that many schemes have been proposed to create a gap in graphene: these suggestions include etching extended graphene flakes into nanoribbons, ${ }^{5,6}$ or by considering bilayer graphene in a transverse electric field, ${ }^{7,8}$ or by using an external periodic potential to modify the electronic properties so that a gap is formed. The external periodic potential may be caused by a number of agents, such as periodic gates ${ }^{9,10}$ or strain, ${ }^{11}$ or adsorption of adatoms in a regular pattern, ${ }^{12,13}$ or, as in this work, by a regular nanoperforation of the pristine graphene sheet; this system will be referred to as graphene antidot lattices (GALs). ${ }^{14}$

The design principle ${ }^{14}$ behind the GAL was inspired by photonic crystals where pass and stop bands for light can be designed by drilling holes in the dielectric medium. GALs (and their constituents, single holes in graphene ${ }^{15}$ ) have been studied theoretically with a large number of methods, ranging from a continuum description and tight-binding methods ${ }^{14,16}$ to fully microscopic DFT calculations. ${ }^{17,18}$ Both electronic $^{19-22}$ and thermal ${ }^{23-25}$ transport properties as well as optical properties ${ }^{26,27}$ have been discussed. Symmetry principles determining the existence or nonexistence of the gap have been outlined. ${ }^{28,29}$ Most important, however, is the recent emergence of experimental techniques by which GALs can be fabricated. These fabrication methods include, e.g., electron-beam etching, ${ }^{30-33}$ etch masks based on selfassembled block co-polymers. ${ }^{34-36}$ nanoimprint technology, ${ }^{37}$ or nanoparticle deposition. ${ }^{38,39}$ Most experimental papers have focused on the structural aspects, but also a few transport experiments have been reported. ${ }^{30,31,33,35,36,39}$ Indeed, transport gaps have been observed but so far they have been associated to disorder induced localization instead of band-structure effects. $^{33,35,36}$ This highlights the importance of studying disorder in GALs: all fabricated structures contain disorder, and one cannot (yet) control the exact geometry of the edges of the etched holes. It is thus vital to examine the robustness of the band gaps against disorder, whether it be structural, geometrical, or chemical. A study of this kind presents a serious computational challenge because the systems fabricated in the laboratory, where unit cells of the order of tens of nanometers can be achieved, are computationally large involving tens of thousands of carbon atoms in the computational cell. Fully microscopic DFT-based methods cannot presently address such systems, and certain compromises must be made.

In this paper, we perform a systematic study of the electronic properties of disordered GALs in the framework of a tight-binding model in a perforated honeycomb lattice of carbon atoms. We consider the most relevant kinds of disorder for these systems, namely a random deviation of the periodicity and of the radii of the nanoholes from the perfect array, as well as the effect of resonant scatterers in the sample (like vacancies, adatoms, etc.) and the effect of noncorrelated and correlated (Gaussian) on-site potentials. Within this scheme, the density of states (DOS) is obtained from a numerical solution of the time-dependent Schrödinger equation (TDSE),${ }^{40}$ and the optical conductivity is calculated by using the Kubo formula for noninteracting electrons. ${ }^{40,41}$ Being interested in the room-temperature electronic structure we do not take into account in our calculations the spin polarization which can arise at the zigzag-type edges at low temperatures. 
The paper is organized as follows. In Sec. II we present the details of the method. The effect of the different kinds of disorder on the DOS and the optical conductivity of a GAL is discussed in Sec. III. Finally, our main conclusions are summarized in Sec. V.

\section{MODEL AND METHOD}

We consider the following real-space tight-binding Hamiltonian for a disordered GAL:

$$
\mathcal{H}=-\sum_{\langle i, j\rangle}\left(t_{i j} c_{i}^{\dagger} c_{j}+\text { H.c. }\right)+\sum_{i} v_{i} c_{i}^{\dagger} c_{i},+\mathcal{H}_{\text {imp }},
$$

where $c_{i}^{\dagger}\left(c_{i}\right)$ creates (annihilates) an electron on site $i$ of the honeycomb graphene lattice, $t_{i j}$ is the nearest-neighbor hopping parameter, and $v_{i}$ is the on-site potential. In our model, the GAL is simulated by the creation of a hexagonal array of circular holes of a given radius $R$, and a separation $P=\sqrt{3} L$ between the centers of two consecutive holes, where $L$ is the side length of the hexagonal unit cell. ${ }^{14} \mathrm{We}$ thus label our GALs with the parameters $\{L, R\}$, in units of the graphene lattice constant $a=\sqrt{3} \tilde{a} \approx 2.46 \AA$, where $\tilde{a} \approx 1.42 \AA$ is the carbon-carbon distance. Another possible notation is $[P, N]$, where $N=\sqrt{3} L-2 R$ is the neck width, defined as the smallest edge-to-edge distance between two neighboring holes in the array. ${ }^{35}$ Deviations of the GALs with respect to perfect periodicity are considered in our calculation in a twofold manner. First, we allow the center of the holes to float with respect to their position in the perfect periodic lattice $(x, y)$ around $\left(x \pm l_{C}, y \pm l_{C}\right)$ [see Fig. 1(a)]. Second, we allow the radius of the holes to randomly shrink or widen within the range $\left[R-r_{R}, R+r_{R}\right]$, as sketched in Fig. 1(b). All along this paper, we will express $l_{C}$ and $r_{R}$ in units of $a$.

The second term on the right of Eq. (1) accounts for a change in the on-site potential of the carbon atoms. A long-range potential for correlated impurities can be modelled with

$$
v_{i}=\sum_{k=1}^{N_{c}} V_{k} \exp \left(-\frac{\left|\mathbf{r}_{i}-\mathbf{r}_{k}\right|^{2}}{2 d^{2}}\right),
$$

where $N_{c}$ is the number of the impurity centers, which are chosen randomly distributed on the carbon atoms, $V_{k}$ is uniformly random in the range $\left[-V_{0}, V_{0}\right]$ and $d$ is interpreted as the effective potential radius. The value of $N_{c}$ is characterized by the ratio $n_{c}=N_{c} / N$, where $N$ is the total number of carbon atoms of the sample. A noncorrelated short-range random potential can be obtained from the above equation with $d \rightarrow 0$, i.e., $v_{i}$ is random and uniformly distributed, independently of each site $i$, in the range $\left[-v_{r},+v_{r}\right]$. The number of sites with nonzero potential $\left(N_{r}\right)$ is characterized as $n_{r}=N_{r} / N$.

We further consider the effect of isolated vacancies in the sample, which can be regarded as an atom (lattice point) with an on-site energy $v_{i} \rightarrow \infty$ or, alternatively, with its hopping amplitudes to other sites being zero. In the numerical simulation, the simplest way to implement a vacancy is to remove the atom at the vacancy site [see Fig. 1(c)].

If additional resonant impurities are present in the sample as, e.g., hydrogen adatoms, their effect is accounted for through
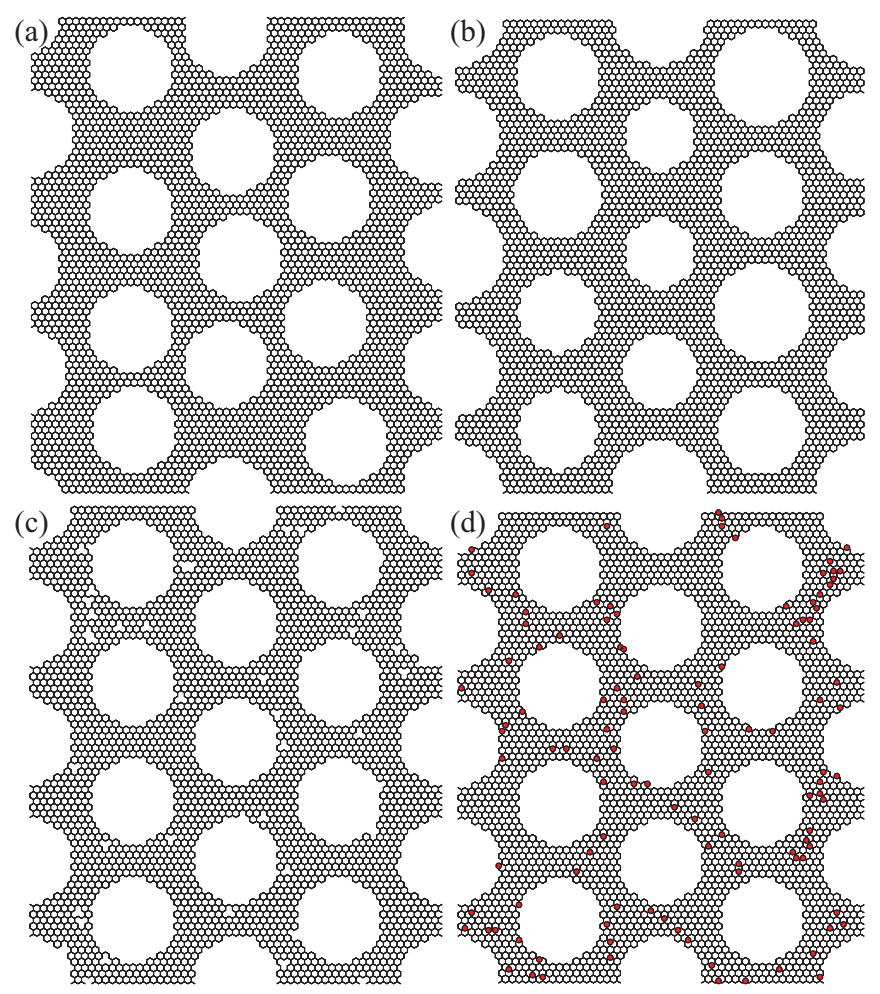

FIG. 1. (Color online) Sketch of the different kinds of disorder considered. (a) The center of the holes is shifted randomly with respect to the original position in the perfect periodic array $(x, y)$ to a new position in the range $\left(x \pm l_{C}, y \pm l_{C}\right)\left(l_{C}=2 a\right)$. (Notice the different relative distance between the holes.) (b) The radius of the holes is randomly shrunk or enlarged within the range $[R-$ $\left.r_{R}, R+r_{R}\right]\left(r_{R}=a\right)$. (Notice the different relative size of the holes.) (c) GAL with additional randomly distributed vacancies, signaled by the missing carbon atoms $\left(n_{x}=1 \%\right)$. (d) GAL with randomly distributed hydrogen adatoms, signaled by the red dots $\left(n_{i}=1.75 \%\right)$. Notice that two other kinds of disorder are considered in the text, namely noncorrelated and correlated long-range (Gaussian) changes in the on-site potentials, which are not sketched in this figure.

the term $\mathcal{H}_{\text {imp }}$ in Eq. (1):

$$
\mathcal{H}_{\text {imp }}=\varepsilon_{d} \sum_{i} d_{i}^{\dagger} d_{i}+V \sum_{i}\left(d_{i}^{\dagger} c_{i}+\text { H.c. }\right)
$$

where $\varepsilon_{d}$ is the on-site potential on the "hydrogen" impurity (to be specific, we will use this terminology although more complicated chemical species can be considered, such as various organic groups) $)^{42}$ and $V$ is the hopping between carbon and hydrogen atoms. ${ }^{40,42,43}$ The spin degree of freedom, which contributes through a degeneracy factor 2 , is omitted for simplicity in Eq. (1). All along this work, we fix the temperature to $T=300 \mathrm{~K}$. We use periodic boundary conditions in the calculations for both the optical conductivity and the density of states, and the size of the system is $6400 \times 6400$ atoms.

Our numerical method is based on an efficient evaluation of the time-evolution operator $e^{-i \mathcal{H} t}$, based on the Chebyshev polynomial representation. ${ }^{40}$ (In fact, any function of $\mathcal{H}$ can be evaluated with this method.) We have thus access to the time-dependent state $|\varphi(t)\rangle \equiv e^{-i \mathcal{H} t}|\varphi\rangle$, where $|\varphi\rangle$ is a random 
superposition of all the basis states in the real space, i.e., ${ }^{40,44}$

$$
|\varphi\rangle=\sum_{i} a_{i} c_{i}^{\dagger}|0\rangle,
$$

$a_{i}$ are random complex numbers normalized as $\sum_{i}\left|a_{i}\right|^{2}=1$, and $|0\rangle$ is the electron vacuum state.

The numerical method has the advantage that an average over different random initial states is not needed. This is because one initial state contains all the eigenstates in the whole spectrum. ${ }^{40,44}$ Furthermore, it is not necessary to average over different realizations of the disorder, because the system contains millions of carbon atoms, and one specific disordered configuration contains a large number of different local configurations. As shown in Ref. 40, the results for different disorder configurations are essentially identical.

Consider first the optical conductivity. We omit in our calculations the $\omega=0$ Drude contribution to the real part of the optical conductivity, so that the regular part can be written as $^{40,45}$

$$
\begin{aligned}
\sigma_{\alpha \beta}(\omega)= & \lim _{\varepsilon \rightarrow 0^{+}} \frac{e^{-\beta \omega}-1}{\omega \Omega} \int_{0}^{\infty} e^{-\varepsilon t} \sin \omega t \\
& \times 2 \operatorname{Im}\left\langle\varphi\left|f(\mathcal{H}) J_{\alpha}(t)[1-f(\mathcal{H})] J_{\beta}\right| \varphi\right\rangle d t,
\end{aligned}
$$

where $\beta=1 / k_{B} T$ is the inverse temperature, $\Omega$ is the sample area, $f(\mathcal{H})=1 /\left[e^{\beta(\mathcal{H}-\mu)}+1\right]$ is the Fermi-Dirac distribution operator, and we use units such that $\hbar=1$. The time-dependent current operator in the $\alpha(=x$ or $y)$ direction is $J_{\alpha}(t)=$ $e^{i \mathcal{H} t} J_{\alpha} e^{-i \mathcal{H} t}$. The Fermi-Dirac distribution operator $f(\mathcal{H})$ is computed with the Chebyshev polynomial representation, as mentioned above.

As the next example, consider the overlap between the time-evolved state $|\varphi(t)\rangle$ and the initial state $|\varphi\rangle$. The Fourier transform of this object yields the DOS of the system as ${ }^{40,44}$

$$
\rho(\varepsilon)=\frac{1}{2 \pi} \int_{-\infty}^{\infty} e^{i \varepsilon t}\langle\varphi \mid \varphi(t)\rangle d t .
$$

Finally, the quasieigenstate $|\Phi(E)\rangle$, which is a superposition of the degenerate eigenstates with the same eigenenergy $E$, is obtained as the Fourier transform of $|\varphi(t)\rangle:^{40}$

$$
|\Phi(E)\rangle=\frac{1}{2 \pi} \int_{-\infty}^{\infty} d t e^{i E t}|\varphi(t)\rangle .
$$

The quasieigenstate is not exactly an energy eigenstate, unless the corresponding eigenstate is not degenerate at energy $E$. However, we can still use the real-space distribution of the amplitude to examine the quasilocalization of the modes. ${ }^{40,41,46}$ Below we display several examples of all these objects.

\section{RESULTS AND DISCUSSION}

In this section we present the results and discuss the effect of the different kinds of disorder introduced in Sec. II, in the
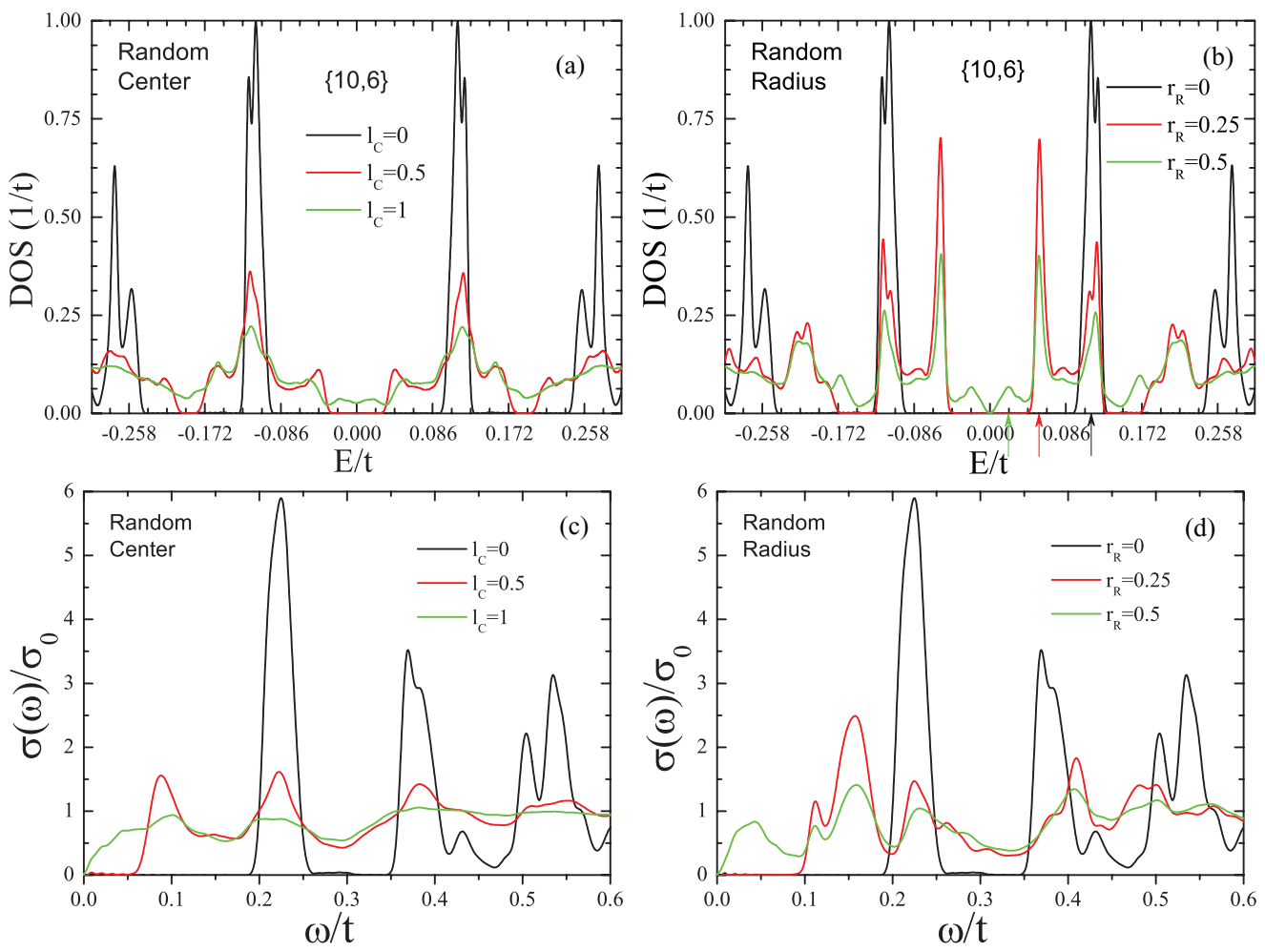

FIG. 2. (Color online) DOS (top panels) and optical conductivity (bottom panels) for a $\{10,6\}$ GAL with geometrical disorder. In the first column we show (a) DOS and (c) $\sigma(\omega)$ for a disordered GAL in which the center of the holes is shifted randomly with respect to the original position in the perfect periodic array within the range $\left(x \pm l_{C}, y \pm l_{C}\right)$, as sketched in Fig. 1(a). The different colors correspond to different values of $l_{C}$ (in units of $a$ ), as denoted in the inset of the figures. In the second column we show (b) DOS and (d) $\sigma(\omega)$ for a GAL where the radius of the holes is randomly shrunk or enlarged within the range $\left[R-r_{R}, R+r_{R}\right]$, as sketched in Fig. 1(b). Different colors correspond to different values of $r_{R}$ (in units of $a$ ). 
DOS and in the optical conductivity of GALs. As discussed in Sec. II and sketched in Fig. 1, we consider three main sources of disorder: geometrical disorder, which is associated to deviations of the GAL from the perfect periodicity; resonant impurities, which can be associated to additional vacancies in the graphene lattice, or to adatoms deposited on the sample; and the effect of on-site potentials which can randomly vary within the sample.

\section{A. Geometrical disorder}

We start by considering the most generic source of disorder in these kinds of systems, which is the geometrical disorder. Uncontrollable fluctuations in the fabrication process lead to irregularities in the resulting antidot lattice, such as changes in the center-to-center distance of the etched holes, or in variations in the size of the holes. Examples of the geometrical disorder in the lattice are sketched in Figs. 1(a) and 1(b), respectively. Let us consider first the effect of a random deviation of the relative distance among the holes on the DOS and $\sigma(\omega)$, as shown in Figs. 2(a) and 2(c) respectively. In Fig. 2(a) we see that, for the perfect periodic array $\left(l_{C}=0\right.$, black line) clear band gaps open up in the spectrum. Notice that the peaked structure of the DOS is due to the set of locally flat bands which appear in the new band structure of the GAL as compared to the spectrum of standard graphene. ${ }^{14}$ If we now allow for a relative displacement among the nanoholes $\left(l_{C} \neq 0\right)$, we observe that the gap shrinks but survives if $l_{C}$ is not too large, as seen by the red line of Fig. 2(a), but eventually the gap closes for some critical value of $l_{C}$, due to the lack of periodicity in the GAL, as is the case shown by the green line of Fig. 2(a) which corresponds to $l_{C}=a$. The disorder affects the optical conductivity in the following manner. As can be seen in Fig. 2(c), for the perfect periodic case $\left(l_{C}=0\right) \sigma(\omega)=0$ up to

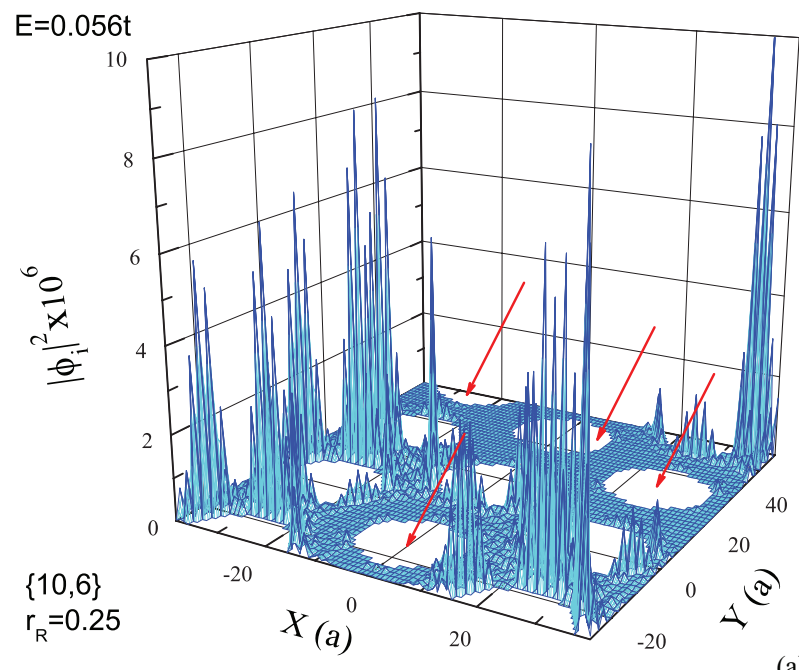

$\omega=\Delta$, where $\Delta \approx 0.2 t$ (for the case considered here) is the gap opened due to the antidot array. Because $\Delta$ decreases as we increase $l_{C}$, the threshold for optical transitions is reduced and for $l_{C}=0.5 a$ we observe a finite optical conductivity for $\omega \gtrsim 0.05 t$. Finally $\sigma(\omega)>0$ at any frequency for an even larger amount of disorder as, e.g., $l_{C}=a$ (green line), for which the gap of the GAL has completely collapsed.

A similar effect on the electronic properties is observed if instead of randomly changing the relative separation between the antidots, their size is varied within some range, as sketched in Fig. 1(b). The results of our simulations for this kind of disorder are shown in Figs. 2(b) and 2(d) for the DOS and optical conductivity, respectively. One observes that the DOS presents an increasing number of peaks as $r_{R}$ is increased. These peaks are associated to states with a large amplitude circling the antidots, and their energy depends on the radius of the antidot. For the pristine GAL $\left(r_{R}=0\right.$, black line) all antidots have the same radius which leads to the peaks at $E / t \sim \pm 0.12$ [signaled by a black arrow in Fig. 2(b)]. The finite width of the peak is due to a coupling between the antidots, and the weak splitting reflects the van Hove singularities at the edges of these quasi-one-dimensional bands. Peaks at higher energies originate from states that are not tightly localized around the antidots, but have a larger amplitude all over the sample. If the radius of the antidots is varied we observe that, apart from the peak discussed above shown by the black arrow, part of the spectral weight is transferred to new peaks that correspond to localized states at different energies, around antidots of different radii. Some examples are shown by the red and green arrows in Fig. 2(b). This behavior is illustrated by the spatial distribution of the quasieigenstates shown in Fig. 3. There we show, for the case of $r_{R}=0.25$, a small section of the lattice studied in our simulations, with a real-space distribution of the amplitude of

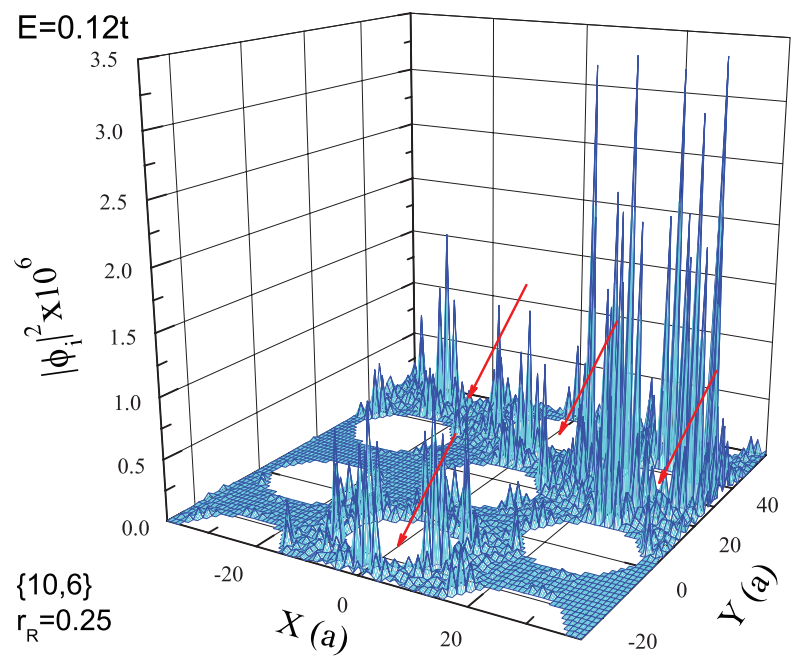

(b)

FIG. 3. (Color online) Distribution of quasieigenstates of a $\{10,6\}$ GAL with geometrical disorder, where the radius of the holes is randomly shrunk or enlarged within the range [5.75,6.25]. The holes with unchanged radius $(R=6)$ are indicated by the red arrows. The quasieigenstates are calculated at energy $E=0.056 t$ and $E=0.12 t$, corresponding to the states at the low-energy peaks marked by the black and red arrows, respectively, in the DOS of Fig. 2(b) $\left(r_{R}=0.25\right)$. The highest contribution to the quasieigensate at $E=0.056 t$ (a) is concentrated around holes with radius $R>6$, for which the first ring of atoms, as compared to perfect GAL with $R=6$, has been removed. The quasieigenstate at $E=0.12 t$ with highest amplitude (b) are localized around the holes with $R=6$ (marked by the red arrows), as in perfect GAL. 
the quasieigenstates $|\Phi(E)|^{2}$. Figure 3(a) shows that, for the energy $E / t \approx 0.056$ [marked by the red arrow in Fig. 2(b)], the large amplitude of the states is around antidots with $R>6$, for which the innermost ring carbon atoms of the antidot has been removed. However, in Fig. 3(b) we see that at an energy corresponding to the first mode of the undistorted lattice $[E / t \approx 0.12$, shown by the black arrow in Fig. 2(b)] the states are localized at the edges of holes with a radius corresponding to a perfect GAL. Those antidots are shown by red arrows in Fig. 3.

The effects of this kind of disorder on the optical conductivity are shown in Fig. 2(d). As $r_{R}$ is increased, we observe optical processes of lower and lower energy contributing to $\sigma(\omega)$, due to optical transitions between the localized states around holes of different sizes. This suggests that photoluminescence spectroscopy can be an useful tool for the characterization of the GALs.

\section{B. Resonant impurities}

The next source of disorder that we consider is the effect of resonant scatterers. Resonant impurities can be understood as vacancy atoms in the sample, or as hydrogen or other organic molecules $\left(\mathrm{CH}_{3}, \mathrm{C}_{2} \mathrm{H}_{5}\right.$, etc. $)$ adsorbates which bind to a single carbon atom, changing its hybridization from $s p^{2}$ to $s p^{3} .^{2,42}$ A sketch of a GAL with a certain amount of vacancies or hydrogen adatoms randomly distributed is shown in Figs. 1(c) and 1(d), respectively. The main effect of the resonant impurities in graphene membranes is the creation of "midgap" states at the Dirac point. ${ }^{41,42}$ Therefore, if some amount of these kinds of impurities is present in the GAL, a zero energy flat impurity band is expected to appear in the middle of the gap. This is indeed what we obtain in our calculations, as can be seen by the $E \approx 0$ peak in the DOS plots shown in Fig. 4. As we discussed in Sec. II, this kind of disorder is accounted for in our calculations through the term $\mathcal{H}_{\text {imp }}$ in Eq. (3), with the band parameters $V \approx 2 t$ and $\varepsilon_{d} \approx-t / 16$, as obtained from $a b$ initio density-functional theory. ${ }^{42}$ The DOS of a GAL with different amounts of vacancies and hydrogen adatoms, randomly distributed, is shown in Figs. 4(a) and 4(b), respectively. We observe that, apart from a slight deviation from the Dirac point $(E=0)$ of the position of the hydrogen adatoms impurity band (due to the finite value of the energy $\varepsilon_{d}$ ), as compared to the $E=0$ energy of the midgap band due to vacancies, the effect of these two kinds of defects in the spectrum is very similar. In the two cases, the quasilocalization of the newly created states leads to an almost flat band which does not affect the rest of the energy spectrum away from the Dirac point (apart from some smearing of the peaks in the DOS).

As a consequence, the main contribution to the optical conductivity is obtained, as in the clean limit, for interband processes with an energy $\omega \approx \Delta$, as can be seen in Figs. 4(c) and $4(\mathrm{~d})$. However, due to the transfer of spectral weight to the midgap states, there is some finite $\sigma(\omega)$ for energies smaller than the threshold defined by the energy gap $\Delta$, with
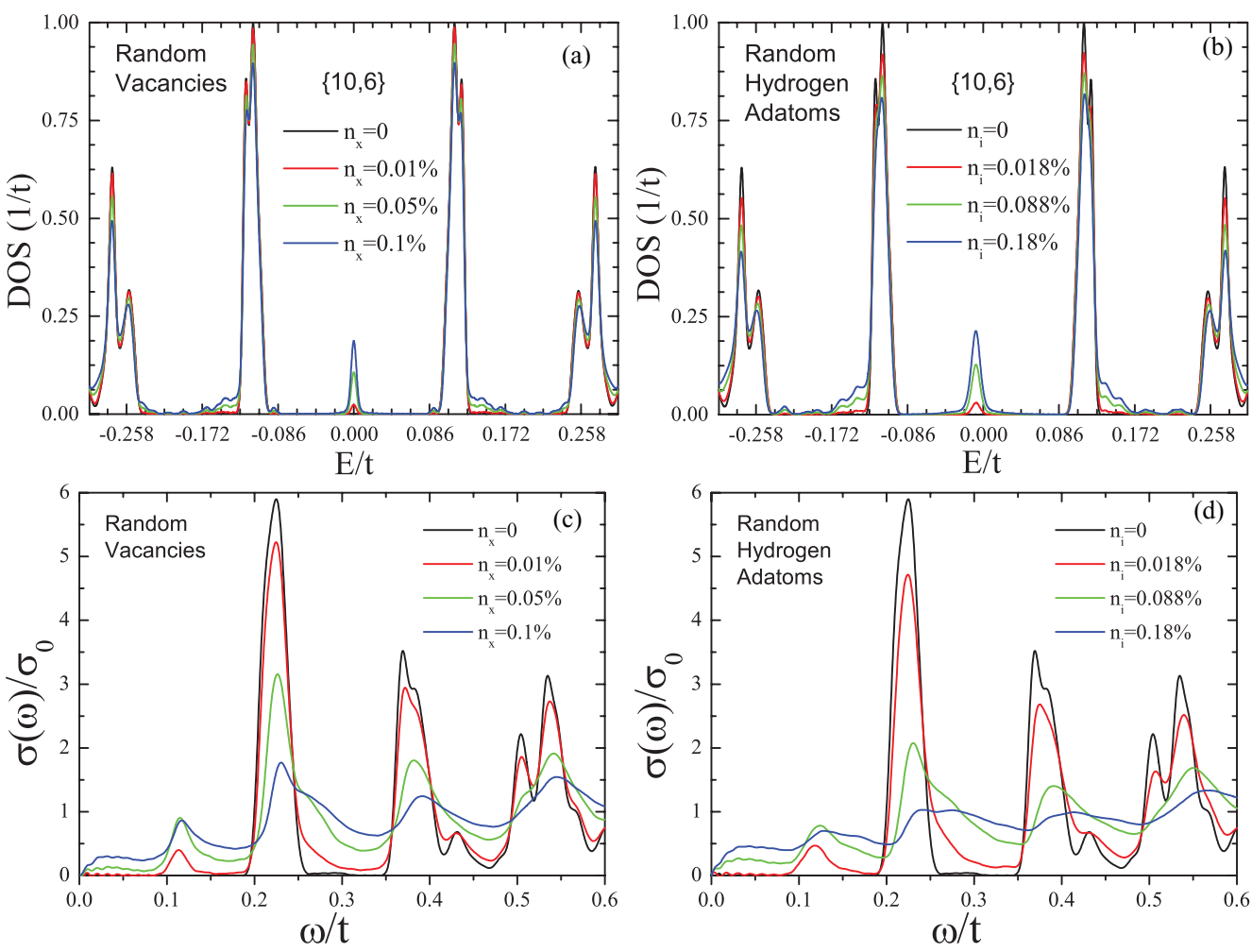

FIG. 4. (Color online) DOS (top panels) and optical conductivity (bottom panels) for a $\{10,6\}$ GAL with resonant impurities. In the first column we show (a) DOS and (c) $\sigma(\omega)$ for a GAL with a random distribution of vacancies, as sketched in Fig. 1(c). The different colors correspond to different amounts of missing dangling bonds, as denoted in the inset of the figures. In the second column we show (b) DOS and (d) $\sigma(\omega)$ for a GAL with hydrogen adatoms, as sketched in Fig. 1(d). Different colors correspond to different percentage of adatoms in the sample. 

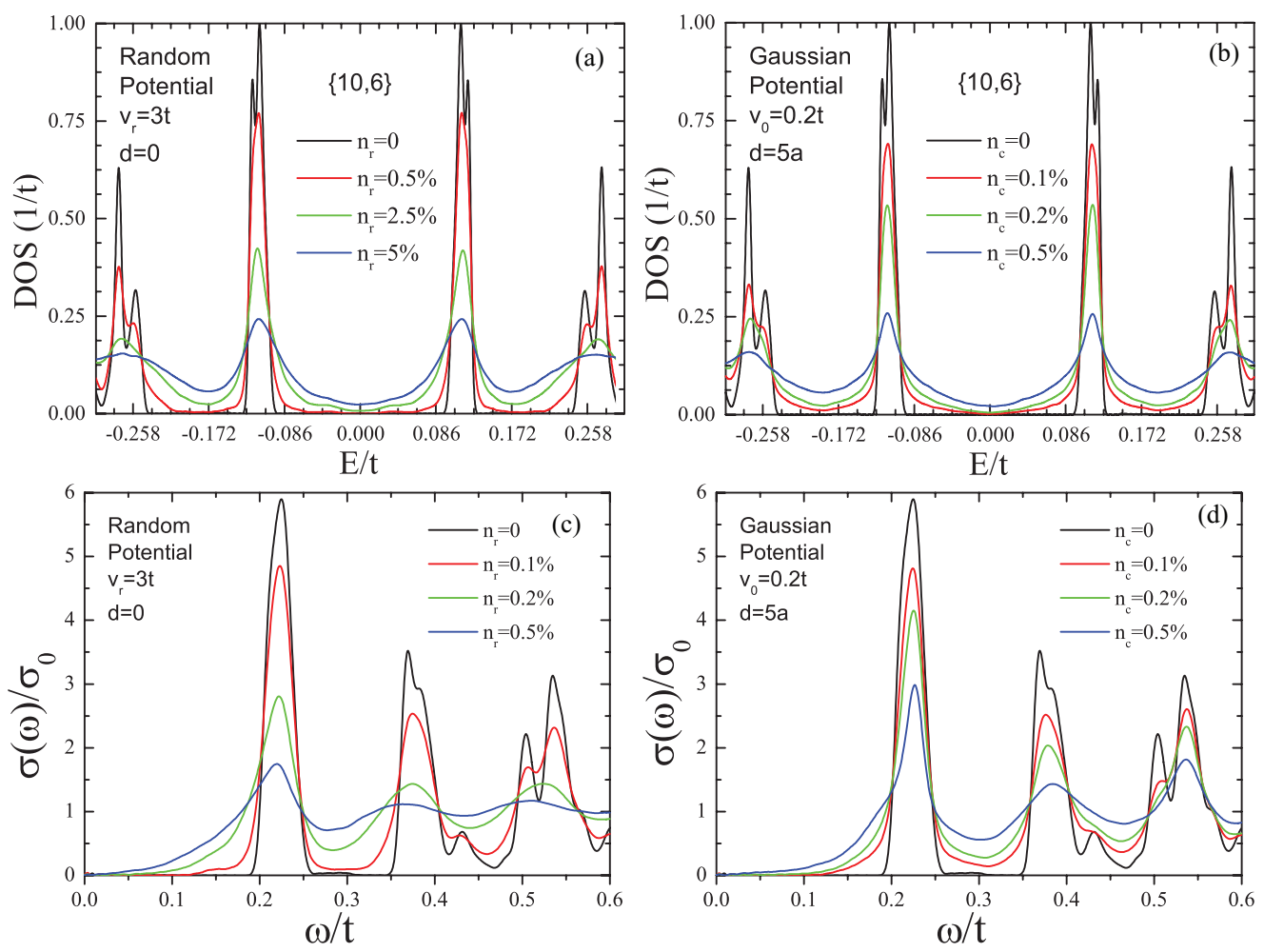

FIG. 5. (Color online) DOS (top panels) and optical conductivity (bottom panels) for a $\{10,6\}$ GAL with on-site potential disorder. In the first column we show (a) DOS and (c) $\sigma(\omega)$ for a GAL with a noncorrelated random distribution of short-range potential, which can take the values within the range $\left[-v_{r}, v_{r}\right]$. The different colors correspond to different concentrations of disorder, as denoted in the inset of the figures. In the second column we show (b) DOS and (d) $\sigma(\omega)$ for a GAL with a long-range Gaussian potential disorder. The potential is given by Eq. (2), where $V_{k}$ is uniformly random in the range between $-V_{0}$ and $V_{0}$, and $d$ is the effective potential radius (see text).

an appreciable peak at $\omega \approx \Delta / 2 \approx 0.1 t$. This contribution is due to the new optical transitions from the impurity band to the conduction band, which are activated for $\omega>\Delta / 2$.

\section{Short- and long-range potential disorder}

Another kind of disorder that can be considered is a shift of the on-site potentials at a given lattice point, which can lead to a local shift of the chemical potential. This contribution is accounted for by means of the second term of the Hamiltonian (1). This kind of disorder can be of extraordinary importance. For example, if the atoms in sublattices $A$ and $B$ have opposite strength of the on-site potential $v_{r}$, then a gap of size $\Delta=2 v_{r}$ is opened in the spectrum. ${ }^{40}$ Here we consider, depending on how the defects are distributed over the lattice sites, a correlated or a noncorrelated disorder. In the case of a short-range and noncorrelated potential disorder, the nonzero on-site potentials are taken to be uniformly randomly distributed over the sample within a range $\left[-v_{r}, v_{r}\right]$. The results for the DOS and the optical conductivity with $v_{r}=3 t$ are shown in Figs. 5(a) and $5(c)$, respectively. We observe a broadening of the peaks in the DOS, accompanied by a transfer of spectral weight to the gapped regions.

Next, consider the long-range correlated disorder given by Eq. (2). In standard graphene, this kind of disorder leads to regions of the graphene membrane where the Dirac point is locally shifted to the electron $\left(V_{k}<0\right)$ or to the hole $\left(V_{k}>0\right)$ side with the same probability. This leads to some finite DOS at zero energy. Our calculations for GALs in the presence of a Gaussian potential disorder are shown in Figs. 5(b) and 5(d), for the DOS and optical conductivity respectively. One observes similar qualitative effects in the spectra as compared to the short-range noncorrelated random potentials. In particular, there is a small but appreciable contribution to the optical conductivity at low frequencies, due to the transfer of states to the gapped region.

\section{A COMMENT ON MAGNETIC PROPERTIES OF DISORDERED GALS}

Recently, magnetoresistance measurements of GALs with hydrogen-terminated and low-defect antidot edges have opened the interest on the magnetic properties of those systems. ${ }^{39}$ Here we comment on the effect of disorder on the magnetism of GALs. Although a complete study of defectinduced magnetism in those systems is beyond the scope of this work (it would require a consideration of electronic correlations which are not included here), we present a qualitative discussion about what one may expect for GALs, based on the existing theoretical work addressing this controversial issue for graphene and other carbon-based materials.

Both density-functional calculations and Hubbard model studies predict a ferromagnetic ground state for zigzag-type grain boundaries in graphene (for reviews, see Chapter 12 of Refs. 2 and 47). This results in a spin splitting of the midgap edge states. ${ }^{15,48}$ However, ferromagnetic ordering does not 
exist for a one-dimensional system (such as graphene edges) at any finite temperatures due to thermal fluctuations. Firstprinciples calculations ${ }^{49}$ give a magnetic correlation length of the order of only $1 \mathrm{~nm}$ at room temperature. Keeping in mind that the magnetic moment in carbon systems is due to $s p$ electrons and is distributed over a broad region (see, e.g., Ref. 50), it seems safe to assume that this spin splitting is unlikely to survive. This issue has been studied for a long time for transition metals by various approaches (see, e.g., Refs. 5155). In some itinerant electron magnets (like chromium) local magnetic moments above magnetic ordering temperature are absent and no signals of spin splitting (an antiferromagnetic shadow gap) is observed whereas for others like iron some smeared spin splitting exists in the paramagnetic phase. The most probable scenario for $s p$-electron magnets is that the Stoner picture is closer to reality: here no spin splitting above the Curie temperature is predicted. ${ }^{56}$

Therefore, irrespective of the controversial issue of ferromagnetism and spin splitting in the ground state (they can be quenched by edge reconstruction, chemical passivation, etc.), magnetism appears irrelevant for the room-temperature electronic properties of the systems studied in this work.

\section{CONCLUSIONS}

In conclusion, we have presented a systematic study of the effect of disorder in GALs. We have used a tight-binding model in a perforated honeycomb lattice of carbon atoms. The DOS has been calculated from a numerical solution of the TDSE, whereas the optical conductivity has been obtained by using the Kubo formula for noninteracting electrons. We have considered the most generic sources of disorder in these kinds of samples: geometrical disorder such as random deviation of the periodicity and of the radii of the nanoholes from the perfect array, as well as the effect of resonant scatterers in the sample (e.g., vacancies, adatoms, etc.) and the effect of noncorrelated and correlated (Gaussian) on-site potentials. In order to have a qualitative understanding of the effect of the different kinds of disorder on the samples, we have applied the method to one representative case, namely a $\{10,6\}$ GAL. However, we emphasize that the employed scheme is completely general and applicable to any set of parameters $\{L, R\}$.

Our results show that the gap is rather robust against geometrical disorder, and only a large deviation of the antidot array from the perfect periodicity leads to a narrowing and eventually closing of the energy gap. We find localized states encircling the antidots, the energy of which depends on the radius of the hole. The presence of additional resonant scatterers, such as vacancies or adatoms, leads to the creation of midgap states. The existence of this impurity band is reflected in the optical conductivity, which now extends to energies smaller than the gap energy $\Delta$, due to disorder activated optical transitions from the impurity band to the conduction band. However, the main contribution to $\sigma(\omega)$ still corresponds to transitions with an energy of the order of $\Delta$. Finally, the presence of noncorrelated or of Gaussian potential disorder leads to a smearing of the peaks in the DOS, as well as to the transfer of spectral weight to the gapped region. Contrary to the effect of resonant scatterers, the presence of potential disorder does not create a zero-energy band with a prominent peak in the DOS at $E=0$, but instead a DOS that grows smoothly as a function of energy within the gapped region. As a consequence, the optical conductivity also grows slowly from 0 until it reaches its maximum contribution at the energy of the gap. Therefore, photoluminescense spectroscopy experiments could be useful for the characterization of the GALs.

\section{ACKNOWLEDGMENTS}

We thank T. G. Pedersen for valuable discussions. The support by the Stichting Fundamenteel Onderzoek der Materie (FOM) and the Netherlands National Computing Facilities Foundation (NCF) are acknowledged. We thank the EUIndia FP-7 collaboration under MONAMI. R.R. acknowledges financial support from the Juan de la Cierva Program (MEC, Spain). The Center for Nanostructured Graphene (CNG) is sponsored by the Danish National Research Foundation, Project DNRF58.

\footnotetext{
*s.yuan@science.ru.nl

†rroldan@icmm.csic.es

${ }^{1}$ A. H. C. Neto, F. Guinea, N. M. R. Peres, K. S. Novoselov, and A. K. Geim, Rev. Mod. Phys 81, 109 (2009).

${ }^{2}$ M. I. Katsnelson, Graphene: Carbon in Two Dimensions (Cambridge University Press, Cambridge, England, 2012).

${ }^{3}$ M. I. Katsnelson, K. S. Novoselov, and A. K. Geim, Nat. Phys. 2 , 620 (2006).

${ }^{4}$ C. W. J. Beenakker, Rev. Mod. Phys. 80, 1337 (2008).

${ }^{5}$ Y.-W. Son, M. L. Cohen, and S. G. Louie, Phys. Rev. Lett. 97, 216803 (2006).

${ }^{6}$ L. Brey and H. A. Fertig, Phys. Rev. B 73, 235411 (2006).

${ }^{7}$ E. McCann, Phys. Rev. B 74, 161403 (2006).

${ }^{8}$ E. V. Castro, K. S. Novoselov, S. V. Morozov, N. M. R. Peres, J. M. B. L. dos Santos, J. Nilsson, F. Guinea, A. K. Geim, and A. H. C. Neto, Phys. Rev. Lett. 99, 216802 (2007).
}

${ }^{9}$ M. Barbier, F. M. Peeters, P. Vasilopoulos, and J. J. M. Pereira, Phys. Rev. B 77, 115446 (2008).

${ }^{10}$ J. G. Pedersen and T. G. Pedersen, Phys. Rev. B 85, 235432 (2012).

${ }^{11}$ T. Low, F. Guinea, and M. I. Katsnelson, Phys. Rev. B 83, 195436 (2011).

${ }^{12}$ J. O. Sofo, A. S. Chaudhari, and G. D. Barber, Phys. Rev. B 75, 153401 (2007).

${ }^{13}$ R. Balog, B. Jorgensen, L. Nilsson, M. Andersen, E. Rienks, M. Bianchi, M. Fanetti, E. Laegsgaard, A. Baraldi, S. Lizzit et al., Nat. Mater. 9, 315 (2010).

${ }^{14}$ T. G. Pedersen, C. Flindt, J. Pedersen, N. A. Mortensen, A.-P. Jauho, and K. Pedersen, Phys. Rev. Lett. 100, 136804 (2008).

${ }^{15}$ J. J. Palacios, J. Fernandez-Rossier, and L. Brey, Phys. Rev. B 77, 195428 (2008).

${ }^{16}$ P. Burset, A. L. Yeyati, L. Brey, and H. A. Fertig, Phys. Rev. B 83, 195434 (2011). 
${ }^{17}$ J. A. Furst, J. G. Pedersen, C. Flindt, N. A. Mortensen, M. Brandbyge, T. G. Pedersen, and A. P. Jauho, New J. Phys. 11, 095020 (2009).

${ }^{18}$ J. A. Furst, T. G. Pedersen, M. Brandbyge, and A. P. Jauho, Phys. Rev. B 80, 115117 (2009).

${ }^{19}$ M. Vanevic, V. M. Stojanovic, and M. Kindermann, Phys. Rev. B 80, 045410 (2009).

${ }^{20}$ Y. P. Bliokh, V. Freilikher, S. Savelev, and F. Nori, Phys. Rev. B 79, 075123 (2009).

${ }^{21}$ L. Rosales, M. Pacheco, Z. Barticevic, A. Leon, A. Latge, and P. A. Orellana, Phys. Rev. B 80, 073402 (2009).

${ }^{22}$ X. H. Zheng, G. R. Zhang, Z. Zeng, V. M. Garcia-Suarez, and C. J. Lambert, Phys. Rev. B 80, 075413 (2009).

${ }^{23}$ T. Gunst, T. Markussen, M. Brandbyge, and A. P. Jauho, Phys. Rev. B 84, 155449 (2011).

${ }^{24}$ Y. H. Yan, Q. F. Liang, H. Zhao, C. Q. Wu, and B. W. Li, Phys. Lett. A 376, 2425 (2012).

${ }^{25}$ P. H. Chang and B. K. Nikolic, Phys. Rev. B 86, 041406 (2012).

${ }^{26}$ T. G. Pedersen, C. Flindt, J. Pedersen, A.-P. Jauho, N. A. Mortensen, and K. Pedersen, Phys. Rev. B 77, 245431 (2008).

${ }^{27}$ T. G. Pedersen, A. P. Jauho, and K. Pedersen, Phys. Rev. B 79, 113406 (2009).

${ }^{28}$ R. Petersen, T. G. Pedersen, and A. P. Jauho, ACS Nano 5, 523 (2011).

${ }^{29}$ F. P. Ouyang, S. G. Peng, Z. F. Liu, and Z. R. Liu, ACS Nano 5, 4023 (2011).

${ }^{30}$ T. Shen, Y. Q. Wu, M. A. Capano, L. P. Rokhinson, L. W. Engel, and P. D. Ye, Appl. Phys. Lett. 93, 122102 (2008).

${ }^{31}$ J. Eroms and D. Weiss, New J. Phys. 11, 095021 (2009).

${ }^{32}$ M. Begliarbekov, O. Sul, J. Santanello, N. Ai, X. Zhang, E.-H. Yang, and S. Strauf, Nano Lett. 11, 1254 (2011).

${ }^{33}$ A. J. M. Gisbers, E. C. Peters, M. Burghard, and K. Kern, Phys. Rev. B 86, 045445 (2012).

${ }^{34}$ M. Bieri, M. Treier, J. Cai, K. Ait-Mansour, P. Ruffieux, O. Groning, P. Groning, M. Kastler, R. Rieger, X. Feng et al., Chem. Commun. 6919 (2009).

${ }^{35}$ J. Bai, X. Zhong, S. Jiang, Y. Huang, and X. Duan, Nat. Nanotechnol. 5, 190 (2010).
${ }^{36}$ M. Kim, N. S. Safron, E. Han, M. S. Arnold, and P. Gopalan, Nano Lett. 10, 1125 (2010).

${ }^{37}$ X. Liang, Y.-S. Jung, S. Wu, A. Ismach, D. L. Olynick, S. Cabrini, and J. Bokor, Nano Lett. 10, 2454 (2010).

${ }^{38}$ A. Sinitskii and J. M. Tour, J. Am. Chem. Soc. 132, 14730 (2010).

${ }^{39}$ T. Shimizu, J. Nakamura, K. Tada, Y. Yagi, and J. Haruyama, Appl. Phys. Lett. 100, 023104 (2012).

${ }^{40}$ S. Yuan, H. De Raedt, and M. I. Katsnelson, Phys. Rev. B 82, 115448 (2010)

${ }^{41}$ S. Yuan, R. Roldán, H. De Raedt, and M. I. Katsnelson, Phys. Rev. B 84, 195418 (2011).

${ }^{42}$ T. O. Wehling, S. Yuan, A. I. Lichtenstein, A. K. Geim, and M. I. Katsnelson, Phys. Rev. Lett. 105, 056802 (2010).

${ }^{43}$ J. P. Robinson, H. Schomerus, L. Oroszlány, and V. I. Fal'ko, Phys. Rev. Lett. 101, 196803 (2008).

${ }^{44}$ A. Hams and H. De Raedt, Phys. Rev. E 62, 4365 (2000).

${ }^{45}$ A. Ishihara, Statistical Physics (Academic, New York, 1971).

${ }^{46} \mathrm{~S}$. Yuan, T. O. Wehling, A. I. Lichtenstein, and M. I. Katsnelson, Phys. Rev. Lett. 109, 156601 (2012).

${ }^{47}$ O. V. Yazyev, Rep. Prog. Phys. 73, 056501 (2010).

${ }^{48}$ J. Fernández-Rossier and J. J. Palacios, Phys. Rev. Lett. 99, 177204 (2007).

${ }^{49}$ O. V. Yazyev and M. I. Katsnelson, Phys. Rev. Lett. 100, 047209 (2008).

${ }^{50}$ D. W. Boukhvalov and M. I. Katsnelson, Eur. Phys. J. B 68, 529 (2009).

${ }^{51}$ V. Heine, J. H. Samson, and C. M. N. Nex, J. Phys. F: Metal Phys. 11, 2645 (1981).

${ }^{52}$ T. Moriya, Spin Fluctuations in Itinerant Electron Magnetism (Springer, Berlin, 1985).

${ }^{53}$ J. Staunton, B. L. Gyorffy, A. J. Pindor, G. M. Stocks, and W. H., J. Phys. F: Metal Phys. 15, 1387 (1985).

${ }^{54}$ S. V. Vonsovsky and M. I. Katsnelson, Physica B 159, 61 (1989).

${ }^{55}$ A. I. Lichtenstein, M. I. Katsnelson, and G. Kotliar, Phys. Rev. Lett. 87, 067205 (2001).

${ }^{56}$ D. M. Edwards and M. I. Katsnelson, J. Phys.: Condens. Matter 18, 7209 (2006). 